\title{
DESENVOLVIMENTO DE PROJETOS NA ÁREA DE QUÍMICA ANALÍTICA POR MEIO DA APRENDIZAGEM BASEADA EM DESAFIOS
}

\author{
DOI: 10.37702/2175-957X.COBENGE.2021.3368
}

Valeska Soares Aguiar - valeska.saguiar@gmail.com

Centro Universitário Facens

Avenida Darci Carvalho Dafferner 151

18085-850 - Sorocaba - SP

Isaias Aguiar Goldschmidt - isaias05@gmail.com

Centro Universitário Facens

Avenida Darci Carvalho Dafferner 151

18085-850 - Sorocaba - SP

Sandra Bizarria Lopes Villanueva - sandra.lopes@facens.br

Centro Universitário Facens

Rod. Sen. José Ermirio de Moraes 1425

18087-125 - Sorocaba - SP

Resumo: Dentro do contexto das novas concepções que cercam as diferentes metodologias de ensino, observamos a presença cada vez mais frequente do aluno como peça central do processo de aprendizagem. Muitas são as metodologias que estimulam o aprendizado discente e, em sua maioria, elas motivam a independência do aluno e o trabalho colaborativo. Na disciplina de Projeto Aplicado à Engenharia Química, vinculada à disciplina de Química Analítica, ambas ministradas no terceiro semestre do curso de bacharelado em Engenharia Química, foi proposto um grande desafio aplicado a diferentes áreas de atuação do futuro engenheiro químico. O desafio formulado pela professora responsável pelas duas disciplinas teve como foco o aprendizado no processo investigativo pela busca por uma solução inovadora para ele. Ou seja, mais importantes que a solução gerada pelos grupos foram as etapas percorridas pelos alunos na criação e no compartilhamento de ideias que estruturaram sua possivel resposta. Por meio da realização de atividades que estimularam a passagem dos alunos por diferentes níveis cognitivos expressos na taxonomia de Bloom, foi possível levantar indícios de alcance da alfabetização científica, uma vez que os alunos puderam aplicar 
diretamente os conhecimentos estudados na disciplina de Química Analítica na resolução do desafio.

Palavras-chave: Aprendizagem baseada em desafios. Alfabetização científica. Química Analítica. 


\section{DESENVOLVIMENTO DE PROJETOS NA ÁREA DE QUÍMICA ANALÍTICA POR MEIO DA APRENDIZAGEM BASEADA EM DESAFIOS}

\section{INTRODUÇÃO}

Um novo perfil de aluno tem sido observado nas décadas iniciais do século XXI. Este aluno nasceu em um meio mais tecnológico, com mais informações e com maior facilidade de acesso a elas, caracterizado pela presença de conhecimentos científicos que evoluem a cada dia. Ao lado deste novo aluno, há um professor formado no século $\mathrm{XX}$, sob as características do ensino informador, e que se encontra em uma zona de conforto que dificilmente é superada. No entanto, tendo em vista este novo perfil de aluno, é evidente a necessidade de mudança nas concepções de ensino desses docentes, de forma que o ensino passe a ter características formadoras, em vez de informadoras.

De acordo com Chassot (2006), o ensino formador é aquele que se distingue do ensino voltado à memorização e, consequentemente, à aprendizagem mecânica. No caso das aulas de Ciências, é o ensino que não foca na memorização de fórmulas prontas. Ou seja, seu objetivo pauta-se em aplicações reais, de forma que o aluno não apenas conheça o conceito oportunamente, em uma avaliação por exemplo, mas saiba aplicá-lo em situações problema reais, de forma que ele se aproprie do conhecimento científico (SASSERON; CARVALHO, 2011).

Para que essa apropriação aconteça da forma mais intensa possível, é extremamente importante que o conteúdo associado ao problema seja relevante ao aluno, ou seja, que seja um conteúdo que o inspire, que o faça acreditar que uma solução é possível e, de forma fundamental, permita que ele aprenda dentro de seu contexto de vida (GUTIÉRREZ et al., 2016).

Assim, muitas metodologias de ensino vêm sendo empregadas em busca dessa apropriação efetiva de conhecimento e, consequentemente, no alcance das competências e habilidades definidas e esperadas para um aluno de Engenharia Química, no caso deste trabalho. Tais metodologias consistem, principalmente, na aprendizagem baseada em equipes, na aprendizagem baseada em problemas e na aprendizagem baseada em projetos (DEVEGILI; LAWALL, 2019; DUMINELLI et al., 2019; LIMA; ARENAS; PASSOS, 2018; MACHADO; BOHM; MORAES, 2018; AZEVEDO; PACHECO; SANTOS, 2019; GEITHNER; POLLASTRO, 2016; SILVA; CASTRO; SALES, 2018; MARQUES; XAVIER, 2019; OLIVEIRA et al., 2020; STOFFEL; PIEMOLINI-BARRETO; SILVA, 2020; VARGAS et al., 2020). Uma metodologia que vem sendo difundida de forma cada vez mais frequente é aquela baseada em desafios (MORESI et al., 2019).

Nesta última modalidade de metodologia de ensino, tem-se uma ampliação da visão associada à resolução de um problema específico (aprendizagem baseada em problemas) e à formulação de um projeto específico na busca por resolver um conjunto de problemas (aprendizagem baseada em projetos). Na aprendizagem baseada em desafios, tem-se a proposta de um grande desafio, que poderá ser resolvido de variadas formas e o foco da metodologia não consiste na solução que será gerada, mas no processo, nas etapas e nos obstáculos a serem superados pelos alunos em sua investigação na busca por uma solução. Os alunos podem inclusive não encontrar uma solução plausível para o desafio, pois o foco é o processo, é o trabalho que acontece ao longo do semestre, na busca pela construção da solução, com a mediação do professor (LOVATO et al., 2018; DUARTE, 2018). 
Nesse processo de busca pelos conhecimentos científicos e sua estruturação para elaborar uma solução possível, o desafio deve apresentar como característica principal o pertencimento à realidade do aluno. $O$ aluno deve se sentir livre para tomar as decisões que considera cabíveis na passagem das etapas desse processo e o professor deve atuar como facilitador de todo o processo, à medida que auxilia o aluno a avançar em busca de uma solução inovadora (LOVATO et al., 2018; GUTIÉRREZ et al., 2016).

Com isso, é possível observar uma relação direta entre a metodologia proposta neste trabalho e o processo de alfabetização científica no Ensino Superior, uma vez que os alunos tiveram a oportunidade de aplicar os conhecimentos científicos associados à análises químicas clássicas, estudadas na disciplina de Química Analítica, nas etapas estruturais de uma solução para um desafio contextualizado à realidade deles. No caso deste trabalho, o desafio proposto consistiu na busca por soluções com características sustentáveis para o problema de tratamento de efluentes industriais. Nesse processo, os alunos deveriam incluir e vincular ao menos um tipo de análise química clássica que pudesse corroborar com a solução construída. É importante ressaltar que o desafio foi trabalhado em uma disciplina que aconteceu de forma paralela à disciplina de Química Analítica, chamada Projeto Aplicado à Engenharia Química, já destinada para o desenvolvimento de projetos aplicados dentro do contexto de atuação da Engenharia Química, podendo ser empregadas diferentes metodologias ativas de ensino e de aprendizagem sendo que, no caso do trabalho aqui exposto, foi empregada a aprendizagem baseada em desafios.

\section{REFERENCIAL TEÓRICO}

A alfabetização científica pode ser compreendida como o processo pelo qual os alunos se apropriam do conhecimento científico por meio da visualização de sua aplicação prática e real. De acordo com Fourez (1997), o conhecimento científico pode ser entendido como um conteúdo que apresenta utilidade ao aluno, daí a importância de ser estudado dentro de um determinado contexto. Para Chassot (2006), a percepção contextualizada do conhecimento científico é fundamental para a construção de um perfil crítico em um aluno, que não apenas reconhece os problemas, mas sabe resolvê-los enxergando a Ciência aplicada a eles.

A formação de um aluno cidadão e crítico pode ser alcançada por meio da aprendizagem baseada em desafios, metodologia aplicada e explicitada neste trabalho. Como meio de análise do favorecimento do processo de aprendizagem e das condições propícias para a alfabetização científica, as etapas de apresentação parcial e final do trabalho desenvolvido foram analisadas de acordo com a taxonomia de Bloom.

De acordo com a teoria desenvolvida por Bloom e colaboradores (BLOOM; KRATHWOHL, 1956) na década de 1950, para que se tenha efetivação da aprendizagem discente, os alunos precisam alcançar níveis cada vez mais altos em termos de cognição. Dessa forma, houve a definição de seis níveis dentro do domínio cognitivo estabelecido por Bloom, sendo eles 1) Conhecimento, 2) Compreensão, 3) Aplicação, 4) Análise, 5) Síntese, e 6) Avaliação (FERRAZ; BELHOT, 2010). Ao longo da segunda metade do século $X X$, aconteceram reformulações na taxonomia, cujos níveis passaram a ser denominados de acordo com os verbos de ação que os descrevem. Além disso, os dois últimos níveis trocaram de posição, pois foi observado que a síntese ou criação pode ser considerada o nível de mais elevada cognição, sendo caracterizado por momentos de transcendência do aluno pelo conhecimento. Assim, as seis categorias de Bloom foram denominadas da seguinte forma 1) Conhecer, 2) Entender, 3) Aplicar, 4) Analisar, 5) 
Avaliar, e 6) Criar (BLOOM; HASTINGS; MADAUS, 1971; ANDERSON, 1999; KRATHWOHL, 2002). O primeiro nível cognitivo que se relaciona à ação "conhecer" se associa aos eventos de memorização e, consequentemente, de aprendizagem mecânica, que leva ao aprendizado instantâneo, útil apenas para o momento da avaliação. Já o nível relacionado à compreensão é de ordem superior uma vez que já permite que outros conhecimentos inerentes ao aluno sejam resgatados na ânsia de interpretar o novo que lhe é apresentado. Ao se permitir "aplicar", o aluno não apenas interpreta o conhecimento, como também o compreende em uma esfera mais ampla, aplicando-o em situações com problemas reais. É a partir do nível da aplicação que podemos visualizar a presença de situações problema contribuindo para o favorecimento do processo de aprendizagem. $\mathrm{Na}$ fase da análise, outras possibilidades de resolução podem ser pensadas, assim como no estágio da avaliação, em que o aluno pode desenvolver seu senso crítico sobre a estrutura pensada e elaborada na resolução de determinado problema. O último nível e com características intensas de superação de aprendizado é aquele que se refere à capacidade de o aluno criar um novo conhecimento a partir daqueles que ele já conhecia e daqueles que foram trabalhados concomitantemente à apresentação do problema. Ao criar, pode-se afirmar que o processo de aprendizagem foi não apenas favorecido como também e, mais importante, efetivado diante das condições colocadas ao aluno.

Os estudos iniciais acerca da taxonomia levavam a acreditar que o aluno deveria ser estimulado por meio de aulas e atividades práticas a passar por cada um dos níveis cognitivos de Bloom, de forma linear, na sequência descrita anteriormente. O conceito das habilidades a serem desenvolvidas na passagem do aluno por cada nível cognitivo encontra-se no Quadro 1.

Quadro 1 - Habilidades a serem desenvolvidas em cada categoria da taxonomia de Bloom

\begin{tabular}{|c|c|c|}
\hline Categoria original & Categoria revisada & Habilidade desenvolvida \\
\hline 1. Conhecimento & 1. Lembrar & Lembrar informações \\
\hline 2. Compreensão & 2. Entender & Compreender o conteúdo de forma a interpretá-lo \\
\hline 3. Aplicação & 3. Aplicar & Usar o conhecimento compreendido em uma situação-problema \\
\hline 4. Análise & 4. Analisar & Ser capaz de estabelecer relações \\
\hline 5. Síntese & 6. Criar & Criar algo novo após o estabelecimento das relações \\
\hline 6. Avaliação & 5. Avaliar & Posicionar-se criticamente diante do que foi criado \\
\hline
\end{tabular}

Fonte: Autoria própria (2021).

Sabe-se que a sequência de passagem de um aluno pelos níveis cognitivos da taxonomia não acontece de forma linear. Diferentes tipos de atividades práticas estimulam o trânsito do aluno pelos níveis a depender de suas concepções prévias, de sua motivação intrínseca e do contexto em que acontece esse estímulo. Assim, o aluno pode se encontrar em mais de um nível cognitivo simultaneamente, uma vez que o processo de aprendizagem é individual e dependente de fatores internos e externos ao aluno.

Ao permitir que o aluno alcance níveis superiores do desenvolvimento cognitivo na pirâmide de Bloom, é possível afirmar que processos de apropriação efetiva do conhecimento foram acionados. Os indícios de ações como analisar, avaliar e criar permitem afirmar que o conhecimento científico pôde ser (re)construído, mediante concepções alternativas dos alunos e facilitação do professor nesse processo de aprendizagem.

Com isso, sabendo que a alfabetização científica pode ser entendida como um processo em que o aluno é capaz de aplicar, analisar, avaliar e criar conhecimento científico dentro de situações contextualizadas próximas a sua realidade, é possível estabelecer uma relação direta entre tal processo e a taxonomia de Bloom, empregada como referencial teórico neste trabalho. 


\section{METODOLOGIA}

A metodologia da aprendizagem baseada em desafios foi aplicada em uma turma do terceiro semestre do curso de Engenharia Química de uma instituição de ensino superior privada, localizada no interior do estado de São Paulo, no primeiro semestre do ano de 2020. A disciplina em que tal metodologia pôde ser desenvolvida, Projeto Aplicado à Engenharia Química, é voltada para a elaboração de projetos por alunos trabalhando em equipes com cinco a dez membros, dependendo da quantidade de alunos por turma. Em cada semestre em que é aplicada, esta disciplina vincula-se a uma disciplina específica da matriz curricular do curso. No caso desta especificamente, o vínculo aconteceu com a disciplina de Química Analítica, que procura abordar as características e os cálculos envolvidos em análises clássicas, tanto qualitativas quanto quantitativas.

Tendo em vista que um desafio deve ser concebido como um problema ou um conjunto de problemas de grande abrangência e deve estar contextualizado à realidade do aluno, o desafio proposto neste trabalho teve como assunto a contaminação das águas, devido ao grande problema relatado pelos órgãos da imprensa em janeiro de 2020 no estado do Rio de Janeiro, em que grande parte da população sofreu com o abastecimento de água, uma vez que ela chegava às torneiras das casas turva e com sinais evidentes de contaminação.

Assim, a partir desse problema é que o tema e o desafio foram construídos e propostos aos grupos de alunos. A delimitação do tema dentro do assunto consistiu em abordar a água de efluentes industriais e, de forma mais específica, o desafio foi transmitido aos alunos por meio da seguinte pergunta: "Como solucionar a contaminação de água por efluentes industriais provenientes das indústrias químicas?".

A definição do campo industrial a ser investigado na busca de uma ou mais soluções para o problema, envolvendo aplicações de análises clássicas estudadas em Química Analítica, aconteceu por grupo. Ou seja, cada grupo desenvolveu um projeto para resolver o mesmo desafio em um determinado campo industrial. Como havia cerca de 70 alunos considerando alunos dos turnos da manhã e da noite, foram formados 12 grupos contendo 5 a 6 membros. Os campos industriais explorados para 0 desenvolvimento dos projetos por cada um dos grupos encontra-se no Quadro 2.

Quadro 2 - Campos industriais explorados pelos grupos no desafio
\begin{tabular}{|c|c|}
\hline Grupo & Campo industrial abordado no desafio \\
\hline 1 & Indústria petroquímica (petróleo e gás) \\
\hline 2 & Indústria agroquímica \\
\hline 3 & Indústria farmacêutica \\
\hline 4 & Indústria de polímeros \\
\hline 5 & Indústria de tintas \\
\hline 6 & Indústria têxtil \\
\hline 7 & Indústria alimentícia \\
\hline 8 & Indústria de domissanitários \\
\hline 9 & Indústria de cosméticos \\
\hline 10 & Indústria do papel \\
\hline 11 & Indústria metalúrgica \\
\hline 12 & Indústria de galvanoplastia \\
\hline
\end{tabular}

Fonte: Autoria própria (2021).

Os grupos puderam escolher o campo industrial que tinham maior interesse e, muitas vezes, proximidade pessoal e/ou profissional. O projeto de cada grupo pôde ser 
acompanhado por meio de relatórios mensais que versaram sobre 1) definição da proposta de solução (ocorrida de forma abrangente, para que os alunos sentissem a presença da possibilidade da flexibilidade em mudar de rota caso considerassem necessário), 2) introdução e revisão da literatura, 3) metodologia, 4) orçamento, gerenciamento de resíduos e resultados esperados, e 5) considerações finais. É importante ressaltar neste momento que, devido à pandemia que chegou ao território brasileiro em meados de fevereiro de 2020, os alunos tiveram apenas quatro semanas de aulas no modelo presencial e, logo na sequência, eles passaram a assistir as aulas e a desenvolver o projeto no modelo remoto. Sendo assim, o projeto aplicado transformou-se em projeto de pesquisa, em que os alunos não aplicaram na prática a metodologia, nem obtiveram resultados concretos em termos físicos, mas puderam simular um conjunto de métodos a ser aplicado experimentalmente, bem como levantar um orçamento real para desenvolver o projeto e delinear os resultados possíveis que seriam esperados a partir da aplicação do projeto. Um dos pontos críticos e extremamente importante nesse levantamento foi a pesquisa sobre os resíduos que seriam gerados no tratamento proposto pelo grupo e sobre os meios possíveis de tratamento para estes. Aqui, podemos destacar uma das características fundamentais que o projeto deveria apresentar, que consistiu no viés sustentável do processo, incluindo a arguição sobre os Objetivos de Desenvolvimento Sustentável (ODS) previstos na agenda 2030 (ONU, 2015).

O outro meio de acompanhamento e avaliação dos projetos aconteceu pela apresentação oral do projeto parcial e do projeto finalizado, ou seja, essa apresentação aconteceu em dois momentos distintos e definidos do semestre. O primeiro momento ocorreu no levantamento e elaboração da metodologia, sem esquecer da presença obrigatória da relação com as análises químicas. E o segundo momento ocorreu no último mês de desenvolvimento do projeto, em que este foi finalizado pelos grupos. Tanto o primeiro quanto o segundo momentos tiveram que ser (re)pensados devido à pandemia $\mathrm{e}$ às limitações que ela trouxe quanto ao ensino telepresencial. Assim, a alternativa empregada foi a produção de um vídeo pitch pelos grupos para cada um desses dois momentos fundamentais para a orientação e para a avaliação dos trabalhos.

\section{RESULTADOS}

Na primeira aula da disciplina de Projeto Aplicado à Engenharia Química, foi aberta uma discussão sobre os principais problemas que estavam sendo enfrentados no país desde o final de 2019 e o início de 2020. O brainstorming estabelecido entre os alunos foi muito produtivo, pois eles puderam se expressar livremente sobre todos os temas que já tinham lido ou visto em algum meio midiático. E dentro desse compartilhamento de ideias, alguns citaram o problema que a cidade do Rio de Janeiro estava passando, momento que foi utilizado para estabelecer a relação com o desafio que seria proposto pela professora responsável.

A receptividade dos alunos foi muito positiva, eles se mostraram interessados em discutir assuntos atuais e em fornecer a própria opinião, e curiosos em saber de que forma uma notícia recente estaria relacionada à disciplina. Este primeiro passo foi muito importante para fornecer o contexto propício para que os alunos compreendessem o desafio e se sentissem estimulados em desenvolver e propor soluções.

As soluções criadas pela maioria dos grupos foram bastante inovadoras e em todas houve a preocupação de incluir uma técnica clássica analítica, com a principal função de monitorar qualitativa ou quantitativamente um contaminante presente no efluente 
industrial. O Quadro 3 reúne as soluções pensadas para o tratamento de cada efluente industrial propostas pelos grupos.

Quadro 3 - Soluções propostas pelos grupos para o desafio

\begin{tabular}{|c|c|c|}
\hline Grupo & Campo industrial abordado no desafio & Tipos de tratamento sustentável \\
\hline 1 & Indústria petroquímica (petróleo e gás) & Uso de carvão ativado e zeólitas \\
\hline 2 & Indústria agroquímica & Uso de biomassa (casca de banana) \\
\hline 3 & Indústria farmacêutica & Uso de biomassa (bagaço de cana-de-açúcar) \\
\hline 4 & Indústria de polímeros & Uso de coagulante (folhas de Aloe vera) \\
\hline 5 & Indústria de tintas & Uso de biomassa (fibra de coco verde) \\
\hline 6 & Indústria têxtil & Uso de carvão ativado \\
\hline 7 & Indústria alimentícia & Uso de coagulação e floculação \\
\hline 8 & Indústria de domissanitários & maracujá) \\
\hline 9 & Indústria de cosméticos & Uso de biomassa (casca de banana, de uva e de \\
\hline 10 & Indústria do papel & Uso de tratamento biológico \\
\hline 11 & Indústria metalúrgica & Uso de fitorremediação \\
\hline 12 & Indústria de galvanoplastia & Uso de semente de moringa \\
\hline
\end{tabular}

Fonte: Autoria própria (2021).

Analisando o Quadro 3, é possível observar que a maioria dos grupos optou por alternativas sustentáveis de tratamento tendo como base processos de adsorção física usando bioadsorventes. Mesmo os grupos que sugeriram opções recorrentes no campo de remoção de particulados de determinados tipos de amostras, como o emprego do carvão ativado, desenvolveram o projeto tendo em vista o encaixe do trabalho nos ODS selecionados pelo grupo. E, mais importante que as soluções propostas, foram as etapas que se sucederam antes da apresentação final do projeto. $O$ caminho percorrido pelos grupos de alunos, que foram estimulados a trabalhar colaborativamente, foram fundamentais para o enriquecimento da bagagem conceitual e, essencialmente, para que ocorresse a (re)construção do conhecimento científico.

Além dos relatórios de acompanhamento, o primeiro vídeo pitch apresentado no semestre permitiu que os alunos organizassem suas pesquisas iniciais para elaborar a revisão da literatura sobre o tema e sobre alguns métodos que estavam em fase de seleção. No momento em que eles organizam as informações na forma de apresentação, gravam o vídeo e o apresentam em momento síncrono na aula surgem novas ideias dentro do próprio grupo, pois o conhecimento dos projetos dos demais grupos também contribui para esse processo criativo. Esse é um momento crucial também para a orientação da professora responsável, que pode visualizar de forma mais concreta e clara todas as etapas que estão sendo sugeridas pelo grupo e propor outras, que podem auxiliá-los a trilhar esse caminho em busca da solução inovadora. Nesse primeiro vídeo pitch produzido no semestre em questão, os grupos se detiveram em explicar as referências que tomaram como base para a construção da possível solução e em expor os fluxogramas iniciais que mostraram as primeiras ideias do processo sob o viés experimental. Neste momento, os alunos já deveriam indicar ao menos um tipo de análise química clássica (conteúdo da disciplina vinculada de Química Analítica) e sua importância, na maioria das vezes associada ao controle de contaminantes, dentro do contexto sustentável do processo de tratamento de efluentes proposto.

O segundo e último vídeo pitch produzido na disciplina foi responsável por exibir as principais fontes consultadas e utilizadas como inspiração para definição dos métodos, bem como por expor o levantamento dos custos da pesquisa caso o projeto fosse colocado em prática, o gerenciamento dos resíduos que seriam produzidos com o 
processo de tratamento proposto e os resultados que seriam esperados (vantagens e desvantagens para a indústria escolhida).

Neste segundo momento em que os alunos puderam se expressar oralmente, que aconteceu na Mostra de Projetos em que os vídeos pitch do projeto finalizado de todos os grupos foram apresentados, eles puderam expressar o pensamento crítico desenvolvido ao longo dos meses, estabelecer comparativos em termos de processos de tratamento mais e menos sustentáveis e, acima de tudo, mostraram evidências de domínio do conteúdo científico aplicado a uma situação problema real, o que reflete o processo de alfabetização científica.

A oportunidade que tiveram de transitar pelos níveis mais altos na taxonomia de Bloom com essa atividade estimuladora fez com que competências transversais pudessem ser trabalhadas. Entre tais competências, as principais que puderam ser estimuladas nos alunos consistiram na criticidade, na expressão oral e escrita e no trabalho colaborativo. Um dos diferenciais trazidos por esta metodologia foi a criação de oportunidades para que os alunos trabalhassem por variados caminhos e não apenas um único, geralmente já pré-estabelecido e orientado pelo professor no caso da aprendizagem baseada em problemas (GUTIÉRREZ et al., 2016).

Além disso, foi possível observar na execução dos vídeos o predomínio dos recursos iconográficos em detrimento dos recursos de texto, a organização da apresentação e a concisão e objetividade na explicação do projeto, uma vez que uma das características do vídeo pitch foi o limite dos 5 minutos de apresentação oral. $O$ dinamismo das apresentações reforçou o conteúdo dos projetos apresentados, aumentando a segurança dos próprios alunos sobre as soluções inovadoras que foram propostas.

Após a avaliação dos relatórios de acompanhamento dos projetos e dos vídeos pitch, com apresentação parcial e final do projeto proposto, foi possível elencar contribuições da metodologia da aprendizagem baseada em desafios aplicada na disciplina em questão para os processos de aprendizagem e de alfabetização científica. As contribuições mais evidentes no discurso dos alunos e em suas produções textuais e orais foram 1) o desenvolvimento da consciência discente sobre problemas atuais, 2) o desenvolvimento simultâneo da criatividade e da criticidade diante de uma solução inovadora (alcance dos níveis cognitivos superiores de Bloom), 3) a percepção concreta da importância do trabalho colaborativo no desenvolvimento de projetos, 4) a visualização na prática de campos reais de aplicação do conhecimento científico, 5) o desenvolvimento da competência investigativa do aluno, e 6) a percepção da relevância de conceitos prévios, inerentes ao aluno e que são extremamente importantes para a (re)construção de novos conhecimentos e para a apropriação dos mesmos.

A oportunidade criada de aproximação dos alunos ao seu campo de trabalho pelo processo investigativo e pela busca por soluções inovadoras também se mostrou uma característica muito positiva na metodologia, pois foi a responsável pelo estímulo à esfera motivacional dos alunos. Muitas vezes, de forma inconsciente, eles aplicaram conhecimentos da área sem perceber que estavam em níveis cognitivos muito além dos processos tradicionais de memorização, à base da aprendizagem mecânica, ainda empregada em muitas instituições de ensino.

\section{CONSIDERAÇÕES FINAIS}

A aprendizagem baseada em desafios mostrou ser uma metodologia ativa de ensino com características satisfatórias e adequadas para ser aplicada em disciplinas 
destinadas ao desenvolvimento de projetos. Qualidades desejáveis no campo profissional como liderança, ética, cidadania, competitividade e espírito empreendedor puderam ser estimuladas nos alunos ao longo do semestre.

A proposta de desafios traz uma nova óptica para a aprendizagem baseada em problemas ou em projetos, pois os desafios elucidam mais de um caminho e mais de uma solução possível. A adaptação de meios de tratamento já existentes e a criação de novas propostas produzem as condições necessárias e propícias para os alunos (re)construírem o conhecimento científico, aplicando-o, analisando-o e avaliando-o. Tais ações são difíceis de serem trabalhadas em meios tradicionais de ensino, pois implicam em um esforço conjunto de aliar professor, aluno e o seu entorno. No entanto, após a definição de uma pergunta base de um desafio, temos o contexto ideal para o surgimento e 0 compartilhamento de ideias pelos alunos.

As competências transversais puderam ser trabalhadas de forma intensa e os meios empregados para avaliação dos alunos refletiram o alcance das mesmas, sendo possível afirmar que tais competências contribuíram diretamente para a formação de um aluno cidadão, crítico, consciente (principalmente em termos das concepções relacionadas ao desenvolvimento sustentável) e que se apropriou do conhecimento científico. Ou seja, podemos afirmar que a atividade aqui proposta teve uma contribuição ímpar em dois processos fundamentais em sala de aula, a alfabetização científica e o processo de aprendizagem, em um contexto com grandes limitações, como foi aquele presente no primeiro semestre do ano de 2020, acometido pela pandemia de COVID-19, que exigiu uma (re)invenção de alternativas para os mais variados campos educacionais e para as diferentes metodologias de ensino.

\section{REFERÊNCIAS}

ANDERSON, Lorin W. Rethinking Bloom's Taxonomy: implication for testing and assessment. Columbia: University of South Carolina, 1999.

AZEVEDO, S. B.; PACHECO, V. A.; SANTOS, E. A. Metodologias ativas no ensino superior: percepção de docentes em uma instituição provada do Distrito Federal. Revista Docência do Ensino Superior, Belo Horizonte, v.9, e002573, p. 1-22, 2019.

BLOOM, Benjamin Samuel; HASTINGS, John Thomas; MADAUS, George F. Handbook on formative and summative evaluation of student learning. New York: McGraw-Hill, 1971.

BLOOM, Benjamin Samuel; KRATHWOHL, David R. Taxonomy of educational objectives. New York: David Mckay, 1956.

CHASSOT, Attico. Alfabetização Científica: questões e desafios para a educação. 4. ed. ljuí: Editora UNIJUÍ, 2006.

DEVEGILI, K. L.; LAWALL, I. T. A construção e aplicação de projetos temáticos e as suas contribuições para uma alfabetização científica e tecnológica. Revista Dynamis, Blumenau, v.25, n.2, p. 224-242, 2019.

DUARTE, Verônica Gonçalves. Metodologias ativas e ensino de ciências na educação superior: um estudo a partir da percepção do aluno. 2018. Dissertação (Mestrado) Educação em Ciências. Universidade Federal de Itajubá, Itajubá, 2018. Disponível em: 
https://repositorio.unifei.edu.br/xmlui/bitstream/handle/123456789/1469/dissertacao_2018 085.pdf?sequence=1\&isAllowed=y. Acesso em 29/03/2021.

DUMINELLI, M. V.; REDIVO, T. S.; BARDINI, C.; YAMAGUCHI, C. K. Metodologias ativas e a inovação na aprendizagem no ensino superior. Brazilian Journal of Development, Curitiba, v.5, n.4, p. 3965-3980, 2019.

FERRAZ, A. P. C. M.; BELHOT, R. V. Taxonomia de Bloom: revisão teórica e apresentação das adequações do instrumento para definição de objetivos instrucionais. Gestão \& Produção, São Carlos, v.17, n.2, p. 421-431, 2010.

FOUREZ, G. Alfabetización Científica y Tecnológica. Acerca de las finalidades de la enseñanza de las ciencias. Buenos Aires: Ediciones Colihue, 1997.

GEITHNER, C. A.; POLLASTRO, A. N. Doing peer review and receiving feedback: impact on scientific literacy and writing skills. Advances in Physiology Education, Bethesda, v.40, p. 38-46, 2016.

GUTIÉRREZ, A. K. C.; ARREOLA, D. M.; RANGEL, E. B.; NÁJERA, E. C. C.; SEPÚLVEDA, F. M. C.; TAMAYO, F. H.; RÍOS, J. B.; MASCARÚA, J. A.; ACHEM, J. G.; DELGADO, J. C.; LASCURAIN, J. P. M.; MEDINA, J. C. B.; LÓPEZ, L. G. G.; LIRA, M. E. V.; GARDNER, N. T.; TRUJILLO, O. L.; ENRÍQUEZ, P. A.; ISLAS, R. L.; MARTÍNEZ, R. M. (org.) Modelo Educativo TEC21. Monterrey: Instituto Tecnológico y de Estudios Superiores de Monterrey, 2016.

KRATHWOHL, D. R. A revision of Bloom's taxonomy: an overview. Theory in Practice, Columbus, v.41, n.4, p. 212-218, 2002.

LIMA, F. S. C.; ARENAS, L. T.; PASSOS, C. G. A metodologia de resolução de problemas: uma experiência para o estudo das ligações químicas. Química Nova, São Paulo, v.41, n.4, p. 468-475, 2018.

LOVATO, F. L.; MICHELOTTI, A.; SILVA, C. B.; LORETTO, E. L. S. Metodologias ativas de aprendizagem: uma breve revisão. Acta Scientiae, Canoas, v.20, n.2, p. 154-171, 2018.

MACHADO, R. C. O.; BOHM, G. M. B.; MORAES, E. Aprendizagem baseada em problemas: um estudo de caso na disciplina de tratamento de água, efluente e lodos. Revista Ibero-Americana de Estudos em Educação, Araraquara, v.13, n.4, p. 18661879, 2018.

MARQUES, R.; XAVIER, C. R. Análise da alfabetização científica de estudantes numa sequência didática de educação ambiental no ensino de ciências. Brazilian Journal of Development, Curitiba, v.5, n.4, p. 2595-2612, 2019.

MORESI, E. A. D.; BRAGA FILHO, M. O.; BARBOSA, J. A.; HARTMANN, V. C. Metodologias ativas de ensino e aprendizagem: o emprego da aprendizagem baseada em desafios na elaboração de revisão de literatura. Indagatio Didactica, Aveiro, v.11, n.3, p. 57-77, 2019. 
OLIVEIRA, F. V.; CANDITO, V.; GUERRA, L.; CHITOLINA, M. R. Aprendizagem baseada em problemas por meio da temática Coronavírus: uma proposta para ensino de Química. Interfaces Científicas, Aracaju, v.10, n.1, p. 110-123, 2020.

ONU. Organização das Nações Unidas. Transformando nosso mundo: a agenda 2030 para o desenvolvimento sustentável. 2015. Disponível em: https://brasil.un.org/ptbr/91863-agenda-2030-para-o-desenvolvimento-sustentavel. Acesso em 04/04/2021.

SASSERON, L. H.; CARVALHO, A. M. P. Alfabetização científica: uma revisão bibliográfica. Investigações em Ensino de Ciências, Porto Alegre, v.16, n.1, p. 59-77, 2011.

SILVA, D. O.; CASTRO, J. B.; SALES, G. L. Aprendizagem baseada em projetos: contribuições das tecnologias digitais. Revista de Educação, Ciência e Tecnologia, Canoas, v.7, n.1, p. 1-19, 2018.

STOFFEL, F.; PIEMOLINI-BARRETO, L. T.; SILVA, P. J. Metodologias participativas e aprendizagem baseada em problemas no curso de Engenharia de Alimentos. Revista de Ensino de Engenharia, Brasília, v.39, p. 337-346, 2020.

VARGAS, S.; ZAMIRPOUR, S.; MENON, S.; ROTHMAN, A.; HÄSE, F.; TOMAYOMENDOZA, T.; ROMERO, J.; SIM, S.; MENKE, T.; ASPURU-GUZIK, A. Team-based learning for scientific computing and automated experimentation: visualization of colored reactions. Journal of Chemical Education, Tucson, v.97, n.3, p. 689-694, 2020.

\title{
PROJECT DEVELOPMENT IN THE ANALYTICAL CHEMISTRY AREA THROUGH CHALLENGE-BASED LEARNING
}

\begin{abstract}
Within the context of the new conceptions that surround the different teaching methodologies, we observe the increasing presence of the student as a central part of the learning process. There are many methodologies that encourage student learning and, for the most part, they motivate student independence and collaborative work. In the discipline of Projects Applied to Chemical Engineering, linked to the discipline of Analytical Chemistry, both taught in the third semester of the Bachelor's degree in Chemical Engineering, the future chemical engineer was challenged in different areas of performance. The professor responsible for the disciplines formulated a challenge focused on learning in an investigative process, and the students had to search for an innovative solution for it. Therefore, more important than the solution generated by the groups were the steps taken by the students in creating and sharing ideas that structured their possible response. Through activities that stimulated students to pass through different cognitive levels expressed in Bloom's taxonomy, it was possible to raise evidence of the reach of scientific literacy, since students were able to directly apply the knowledge studied in the discipline of Analytical Chemistry in solving the challenge.
\end{abstract}

Keywords: Challenge-based learning. Scientific literacy. Analytical chemistry. 